\title{
Ankle-brachial blood pressure differences in the beach- chair position of the shoulder surgery
}

\author{
Jae Chan Choi ${ }^{1}$, Jong-Hyuk Lee ${ }^{1}$, Young-Don Lee ${ }^{1}$, Soon Yul Kim ${ }^{1}$, and Sei-Jin Chang ${ }^{2}$ \\ Departments of ${ }^{1}$ Anesthesiology and Pain Medicine, ${ }^{2}$ Preventive Medicine, Yonsei University Wonju College of Medicine, Wonju, Korea
}

Background: During shoulder surgery, blood pressure is frequently measured at the ankle. Anesthetic complications may result when ankle blood pressure is higher than brachial blood pressure and anesthesiologists misinterpret ankle blood pressure as brachial blood pressure. Therefore, we investigated whether ankle blood pressure is significantly higher than brachial blood pressure before anesthesia induction, during induction, after tracheal intubation, before beach chair position, and in the beach chair position.

Methods: Thirty patients requiring general anesthesia for shoulder surgery were included in this study. Ankle and brachial blood pressure were simultaneously measured before induction, during induction, after intubation, before beach chair position, and in the beach chair position.

Results: Ankle blood pressure was higher than brachial blood pressure before induction, during induction, after intubation, before beach chair position, and in the beach chair position. Ankle-brachial blood pressure differences in the beach chair condition were much higher than in four other conditions. The correlation coefficient between mean ankle-brachial blood pressure differences before the beach chair position and mean ankle-brachial blood pressure differences in the beach chair position was 0.616 . Brachial systolic blood pressure could be predicted by regression equations $\left(R^{2}=0.306-0.771\right)$.

Conclusions: These results suggest that anesthesiologists should consider these ankle-brachial blood pressure differences when monitoring anesthesia in the beach chair position. (Korean J Anesthesiol 2012; 63: 515-520)

Key Words: Ankle blood pressure, Ankle-brachial blood pressure index, Beach chair position, Blood pressure, Brachial blood pressure, Shoulder surgery.

Received: March 23, 2012. Revised: 1st, May 3, 2012; 2nd, May 29, 2012; 3rd, June 14, 2012; 4th, June 15, 2012; 5th, July 3, $2012 ; 6$ th, July 4, 2012. Accepted: July 5, 2012.

Corresponding author: Jae Chan Choi, M.D., Ph.D., Department of Anesthesiology and Pain Medicine, Yonsei University Wonju College of Medicine, 162, Ilsan-dong, Wonju 220-701, Korea. Tel: 82-33-762-8402, Fax: 82-33-742-8198, E-mail: jaechan@yonsei.ac.kr

(c) This is an open-access article distributed under the terms of the Creative Commons Attribution Non-Commercial License (http:// creativecommons.org/licenses/by-nc/3.0/), which permits unrestricted non-commercial use, distribution, and reproduction in any medium, provided the original work is properly cited. 


\section{Introduction}

During shoulder surgery, ankle blood pressure is frequently used for monitoring anesthesia in place of brachial blood pressure, as the intravenous line in the unoperated arm or forearm is obstructed by the blood pressure cuff, and this can prevent the administration of intravenous anesthetics. In addition, because chest and brachial sphygmomanometer cuff is tightly compressed by safety belt in order to prevent patient's fall from operating table, brachial blood pressure on some occasions cannot be reliably measured. In this situation, anesthesiologists measure ankle blood pressure using an automatic sphygmomanometer, when the patient's physical status is graded as American Society of Anesthesiologists (ASA) I or II, and without the need for invasive arterial cannulation. In the case that ankle blood pressure can be used in monitoring anesthesia, if anesthesiologists know ankle-brachial blood pressure differences, anesthetics and antihypertensive drugs can be adequately administered to patients. A recent study has shown that inadequate blood pressure monitoring is associated with inadequate intraoperative blood transfusions, vasopressor infusions, and antihypertensive medication administration [1].

Some surgeons favor the beach-chair position for arthroscopic shoulder surgery. Advantages of the beach-chair position are that this position can avoid distortion of the intraarticular anatomy and use the weight of the arm to distract the joint [2-4]. In the beach chair position, blood pressures are usually measured by ankle cuff, because the intravenous line is in the unoperated forearm and non-operative arm is fastened by belt. However, complications including stroke and brain death, loss of vision, and ophthalmoplegia have been reported in shoulder surgery of the beach-chair position $[2,3,5,6]$. It is known that such complications are presumed to be attributable to misinterpretation of the ankle-brachial blood pressure differences [4]. In shoulder operations performed in the beachchair position, anesthesiologists should not regard ankle blood pressure as equal to blood pressure measured at the heart level.

Although blood pressure and heart rate cannot completely assess the depth of anesthesia blood pressure can be used as an indicator of pain during full surgical anesthesia [7]; however, blood pressure and heart rate can vary for other reasons (for example, head up position, hypertension and dehydration). In the practice of anesthesia, anesthesiologists interpret increases in blood pressure as an indicator of the onset of pain if there are no another causes that increase the blood pressure $[8,9]$. Anesthetic complications may result if anesthesiologists misinterpret ankle blood pressure as brachial blood pressure.

In this study, we investigated whether ankle blood pressure is significantly higher than brachial blood pressure before induction of anesthesia, during anesthetic induction, imme- diately after tracheal intubation. Because there is no manuscript that statistically analyzed ankle-brachial blood pressure differences in the beach-chair position, we investigate the anklebrachial blood pressure differences before beach chair position and in the beach chair position.

\section{Materials and Methods}

Thirty-six patients (ASA physical status of I or II), who required general anesthesia for shoulder surgery, were included in this study. However, because brachial blood pressure was not reliably measured in 6 patients, these patients were withdrawn from the study. Therefore, data from 30 patients were used in the analyses (age [yr, $56.80 \pm 9.58$ (SD)], body weight [kg, $62.81 \pm$ 6.76], height [cm, $159.56 \pm 9.58]$, body mass index $\left[\mathrm{kg} / \mathrm{m}^{2}, 24.61\right.$ $\pm 2.42]$, male [ $n=18]$, female [ $n=12]$ ). This study was approved by the Medical Ethics Committee of our hospital and the patients after the study objectives were described prior to surgery. Ankle and brachial blood pressure were simultaneously measured with $12 \mathrm{~cm}$-wide cuffs using a Philips IntelliVue MP40 patient monitor (Phillips, Boeblingen, Germany) and a Philips SureSigns VM6 monitor (Philips, Andover, MA, USA).

Blood pressure and heart rate were measured in the following 5 conditions [before induction condition (BIC), during induction condition (DIC), after intubation condition (AIC), before beach chair condition (BeBeach), and beach chair condition (Beach)]. After patients arrived in the operating room and before anesthetic induction, blood pressure and heart rate in the supine position were measured on the unoperated arm and the same side ankle to the unoperated arm (BIC = before induction condition). Following preoxygenation, anesthesia was induced with $4 \mathrm{mg} /$ $\mathrm{kg}$ thiopental and $0.1 \mu \mathrm{g} / \mathrm{kg} / \mathrm{min}$ remifentanil by infusion. To facilitate orotracheal intubation, $0.8 \mathrm{mg} / \mathrm{kg}$ rocuronium was administered. Anesthesia was maintained with sevoflurane 1.5$2.0 \%$ in a $50 \%$ oxygen and remifentanil infusion, with positive pressure ventilation in a circle system. The administration of anesthetic agents was adjusted, while considering patient age and pain intensity. Blood pressure and heart rate were also measured in the supine position following administration of anesthetic induction agents (DIC $=$ during induction condition). Blood pressure in the DIC was measured during anesthetic induction to assess the difference between ankle and brachial blood pressure in the anesthetic-induced decreased blood pressure state. Immediately after tracheal intubation, blood pressure and heart rate were again measured in the supine position (AIC = after intubation condition). Blood pressure in the AIC was measured to assess the ankle-brachial blood pressure difference in the increased blood pressure state due to intubation-induced pain.

After trachea was intubated and patient's blood pressure was 
stable, immediately before operating table was elevated, blood pressure and heart rate in the supine position were measured in the unoperated arm and ankle (BeBeach = before beach chair condition). In the beach chair position, the operating table was flexed approximately 45 degrees and the patient sat with approximately 45 degrees head up and both stretched legs lay on an horizontal operating table.

In the beach-chair position, blood pressure and heart rate were again measured in the unoperated arm and ankle (Beach = beach chair condition). When the position changed from the supine to the beach chair position, anesthetics and fluid infusion rate were not changed. Ankle and brachial blood pressures in the beach chair position were measured 3 times per patient ( 1 minute, 3 minute, 5 minute after the beach chair position) and average of 3 blood pressure values was calculated.

An ankle-brachial blood pressure index (ABI) is the ratio of the ankle to brachial systolic blood pressure. The ABI $<0.90$ indicates the presence of peripheral arterial disease [10]. We investigated the number of patients with an $\mathrm{ABI}<0.90$ prior to induction. In addition, we investigated the number of the patients with an $\mathrm{ABI}<1$ before induction, indicating that ankle systolic blood pressure was lower than the brachial systolic blood pressure.

To obtain the sample size, we use mean \pm SD of SBP baseline in reference [11] and a clinically significant difference is considered to be $20 \%$. The required sample size for having an $80 \%$ power (ie, $\beta=0.2$ ) at $\alpha=0.05$ can be obtained by normal approximation as $\mathrm{n}=\left(\mathrm{Z}_{\alpha}+\mathrm{Z}_{\beta}\right)^{2} \sigma^{2} /(\varepsilon-\delta)^{2} \approx 30$ [12]. When we consider $10 \%$ drop rate, the sample size needed is 34 . Statistical analysis was performed with PASW Statistics 18 (Predictive
Analytics Software; SPSS Inc., Chicago, Illinois, USA). Ankle blood pressure was compared to brachial blood pressure using the paired t-test before the induction of anesthesia, during anesthetic induction, immediately after tracheal intubation, before the beach position, and in the beach chair position. Ankle-brachial blood pressure differences were compared among these 5 conditions using repeated measures ANOVA. Correlation coefficient was calculated using a correlation analysis and a regression equation was acquired from the regression analysis. All results were considered significant at a threshold of $\mathrm{P}<0.05$.

\section{Results}

Ankle blood pressure was compared to brachial blood pressure using paired t-test in the 30 patients in the five study conditions (BIC, DIC, AIC, BeBeach, and Beach) (Table 1). There were significant systolic and mean ankle-brachial blood pressure differences in the 5 conditions. Diastolic ankle blood pressures were significantly higher than diastolic brachial blood pressures only in the Beach position, while diastolic anklebrachial blood pressure differences were not significantly different in the 4 other conditions except for the Beach.

SABBPD (systolic ankle-brachial blood pressure difference $=$ systolic blood pressure in ankle - systolic blood pressure in brachium) in 30 patients was significantly different among the 5 study conditions according to repeated measures ANOVA (F value $=9.91 .70, \mathrm{P}<0.001)$ (Table 1 and Fig. 1$)$. When the Bonferroni correction was used for multiple comparisons, the SABBPD was significantly higher in the Beach $(33.97 \pm$

Table 1. Ankle Blood Pressure Compared to Brachial Blood Pressure at Several Time Points and Positions $(\mathrm{N}=30)$

\begin{tabular}{|c|c|c|c|c|c|}
\hline & & $\begin{array}{c}\text { Brachial NIBP } \\
\text { (mmHg) }\end{array}$ & $\begin{array}{l}\text { Ankle NIBP } \\
\text { (mmHg) }\end{array}$ & $\begin{array}{l}\text { ABBPD } \\
(\mathrm{mmHg})\end{array}$ & $\mathrm{P}$ value \\
\hline \multirow[t]{3}{*}{ BIC } & SBP & $129.50 \pm 15.90$ & $151.83 \pm 20.29$ & $22.33 \pm 12.11$ & $\mathrm{P}<0.001$ \\
\hline & DBP & $79.07 \pm 11.61$ & $78.63 \pm 9.38$ & $-0.43 \pm 8.39$ & $P=0.779$ \\
\hline & MBP & $95.88 \pm 11.64$ & $103.03 \pm 11.36$ & $7.16 \pm 7.47$ & $\mathrm{P}<0.001$ \\
\hline \multirow[t]{3}{*}{ DIC } & SBP & $108.23 \pm 16.26$ & $126.83 \pm 19.13$ & $18.60 \pm 12.35$ & $\mathrm{P}<0.001$ \\
\hline & DBP & $74.33 \pm 13.51$ & $74.00 \pm 13.09$ & $-0.33 \pm 7.48$ & $P=0.809$ \\
\hline & MBP & $86.63 \pm 13.97$ & $91.61 \pm 13.83$ & $5.98 \pm 7.73$ & $\mathrm{P}<0.001$ \\
\hline \multirow[t]{3}{*}{ AIC } & SBP & $163.07 \pm 21.57$ & $185.53 \pm 27.29$ & $22.47 \pm 15.87$ & $\mathrm{P}<0.001$ \\
\hline & $\mathrm{DBP}$ & $104.07 \pm 17.69$ & $101.03 \pm 16.74$ & $-3.03 \pm 9.29$ & $P=0.084$ \\
\hline & MBP & $123.73 \pm 18.26$ & $129.20 \pm 18.50$ & $5.47 \pm 9.39$ & $P=0.003$ \\
\hline \multirow[t]{3}{*}{ BeBeach } & SBP & $107.20 \pm 22.67$ & $127.77 \pm 19.42$ & $20.57 \pm 12.00$ & $\mathrm{P}<0.001$ \\
\hline & DBP & $73.20 \pm 14.64$ & $74.73 \pm 13.37$ & $1.53 \pm 10.54$ & $P=0.432$ \\
\hline & MBP & $84.53 \pm 16.42$ & $92.41 \pm 14.88$ & $7.88 \pm 9.16$ & $\mathrm{P}<0.001$ \\
\hline \multirow[t]{3}{*}{ Beach } & SBP & $99.57 \pm 17.97$ & $133.77 \pm 17.31$ & $33.97 \pm 10.67$ & $\mathrm{P}<0.001$ \\
\hline & $\mathrm{DBP}$ & $66.73 \pm 15.45$ & $84.73 \pm 11.77$ & $18.00 \pm 8.36$ & $\mathrm{P}<0.001$ \\
\hline & MBP & $77.56 \pm 15.66$ & $101.08 \pm 13.13$ & $23.32 \pm 7.52$ & $\mathrm{P}<0.001$ \\
\hline
\end{tabular}

Data = mean \pm SD. NIBP: noninvasive blood-pressure monitoring, BIC: before induction condition, DIC: during induction condition, AIC: after intubation condition, BeBeach: before beach chair condition, Beach: beach chair condition, SBP: systolic blood pressure, DBP: diastolic blood pressure, MBP: mean blood pressure, ABBPD: ankle-brachial blood pressure difference. 
10.67) than in the 4 other conditions (Beach vs. BIC, $\mathrm{P}=0.003$; Beach vs. DIC, $\mathrm{P}<0.001$; Beach vs. AIC, $\mathrm{P}=0.036$; Beach vs. BeBeach, $\mathrm{P}<0.001$ ). MABBPD (mean ankle-brachial blood pressure difference $=$ mean blood pressure in ankle - mean blood pressure in brachium) in 30 patients was significantly different among the 5 study conditions according to repeated measures ANOVA $(\mathrm{F}$ value $=39.79, \mathrm{P}<0.001)$ (Table 1$)$. When the Bonferroni correction was used for multiple comparisons, the MABBPD was significantly higher in the Beach $(23.32 \pm 7.52)$ than in the 4 other conditions (Beach vs. BIC, $\mathrm{P}<0.001$; Beach vs. DIC, $\mathrm{P}<0.001$; Beach vs. AIC, $\mathrm{P}<0.001$; Beach vs. BeBeach, $\mathrm{P}<0.001)$.

Heart rate (beats/min) was not significantly different between the BeBeach (82.53 \pm 14.50$)$ and Beach (80.03 \pm 14.09$)$.

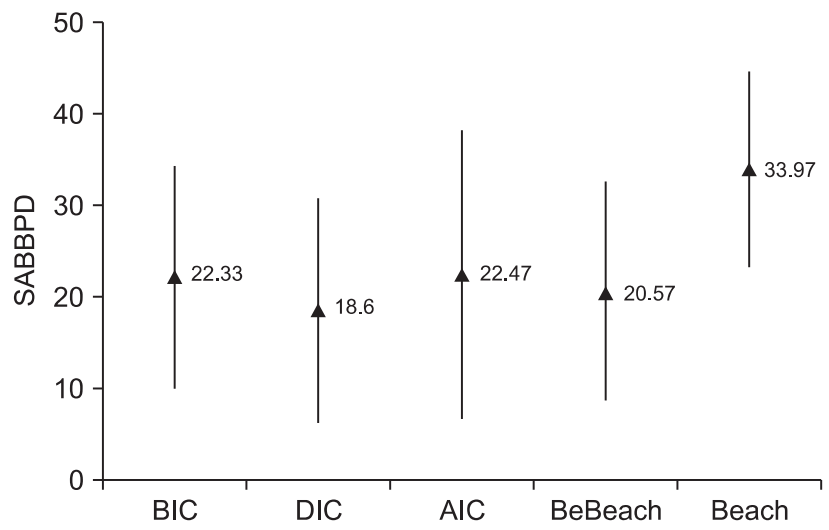

Fig. 1. Systolic ankle-brachial blood pressure differences among the 5 study conditions in 30 patients. The SABBPD (systolic anklebrachial blood pressure difference $=$ systolic blood pressure in ankle - systolic blood pressure in brachium) is significantly higher in the Beach $(33.97 \pm 10.67)$ than in the 4 other conditions (Beach vs. BIC, $\mathrm{P}=0.003$; Beach vs. DIC, $\mathrm{P}<0.001$; Beach vs. AIC, $\mathrm{P}=0.036$; Beach vs. BeBeach, $\mathrm{P}<0.001)$. Also, the SABBPD is significantly higher in the BIC than in the DIC $(\mathrm{P}=0.023)$. BIC: before induction condition, DIC: during induction condition, AIC: after intubation condition, BeBeach: before beach chair condition, Beach: beach chair condition. Triangles are means and whiskers are SDs.
The correlation coefficient $(r)$ between the MABBPD in the BeBeach and the MABBPD in the Beach was $0.616(\mathrm{P}<0.001$, Fig. 2) in 30 patients. This result indicates that the mean anklebrachial blood pressure differences in the BeBeach could predict the mean ankle-brachial blood pressure differences in the Beach. A regression equation was acquired from the regression analysis (Table 2). In the event that brachial systolic blood pressure cannot be measured in the BeBeach and Beach, brachial systolic blood pressure could be inferred from ankle systolic blood pressure using the regression equations described in Table 2. If the SABBPD and MABBPD were measured in the BeBeach, the SABBPD and MABBPD in the Beach could be inferred from the regression equations described in Table 2.

There was no patient with an $\mathrm{ABI}<0.90$ in the BIC, and the number of patients with an $\mathrm{ABI}<1$ was 2 in the BIC. The correlation coefficient $(r)$ between the ABIs in the BeBeach and the ABIs in the Beach was determined using a correlation analysis. The $r$ between the ABIs in the BeBeach and the ABIs in

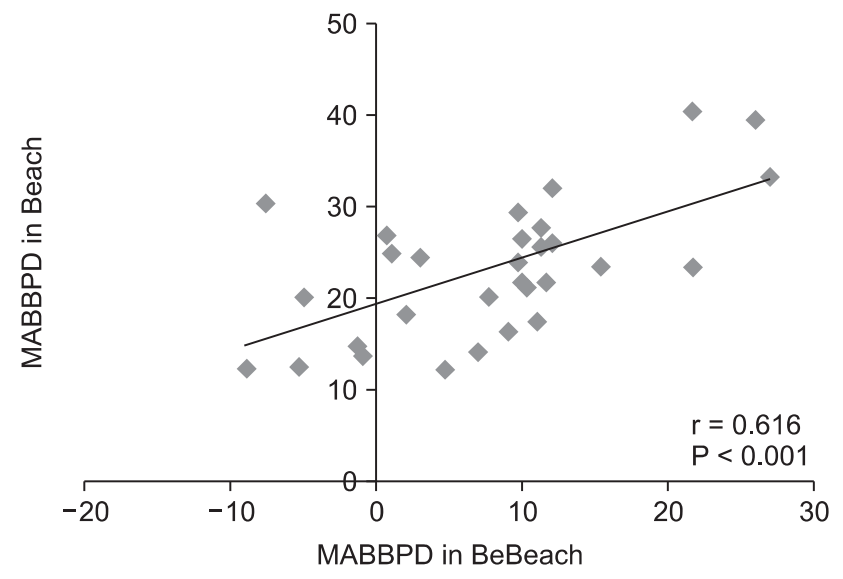

Fig. 2. The correlation between the MABBPD in the BeBeach and the MABBPD in the Beach. MABBPD (mean ankle-brachial blood pressure difference) $=$ mean blood pressure in ankle - mean blood pressure in brachium. BeBeach: before beach chair condition, Beach: beach chair condition, $r$ : correlation coefficient.

Table 2. Regression Equations Acquired from Regression Analysis in the BeBeach and Beach

\begin{tabular}{|c|c|c|}
\hline Regression equation & $\mathrm{R}^{2}$ & $F$ value, $P$ value \\
\hline Brachial SBP in BeBeach $=-19.31+0.99$ ankle SBP in BeBeach & 0.720 & $\mathrm{~F}(1,28)=71.88, \mathrm{P}<0.001$ \\
\hline Brachial MBP in BeBeach $=-19.31+0.99$ ankle MBP in BeBeach & 0.694 & $\mathrm{~F}(1,28)=63.50, \mathrm{P}<0.001$ \\
\hline Brachial SBP in Beach $=-13.77+0.85$ ankle SBP in Beach & 0.669 & $\mathrm{~F}(1,28)=56.47, \mathrm{P}<0.001$ \\
\hline Brachial DBP in Beach $=-27.21+1.11$ ankle DBP in Beach & 0.714 & $\mathrm{~F}(1,28)=69.93, \mathrm{P}<0.001$ \\
\hline Brachial MBP in Beach $=-28.11+1.05$ ankle MBP in Beach & 0.771 & $\mathrm{~F}(1,28)=94.35, \mathrm{P}<0.001$ \\
\hline Brachial SABBPD in Beach $=23.89+0.49$ SABBPD in BeBeach & 0.306 & $\mathrm{~F}(1,28)=12.33, \mathrm{P}=0.002$ \\
\hline Brachial MABBPD in Beach $=19.34+0.51 \mathrm{MABBPD}$ in BeBeach & 0.380 & $\mathrm{~F}(1,28)=17.14, \mathrm{P}<0.001$ \\
\hline
\end{tabular}

SBP: systolic blood pressure, DBP: diastolic blood pressure, MBP: mean blood pressure, BeBeach: before beach chair condition, Beach: beach chair condition, $\mathrm{R}^{2}$ : coefficient of determination. SABBPD (systolic ankle-brachial blood pressure difference) = systolic blood pressure in ankle - systolic blood pressure in brachium, MABBPD (mean ankle-brachial blood pressure difference) $=$ mean blood pressure in ankle - mean blood pressure in brachium. 
the Beach was $0.834(\mathrm{P}<0.001)$. This indicates that if the systolic ankle blood pressure is higher than systolic brachial blood pressure in the BeBeach, systolic ankle blood pressure will be higher than systolic brachial blood pressure in the Beach.

\section{Discussion}

In the present study, ankle blood pressure was higher than brachial blood pressure before the induction of anesthesia, during anesthetic induction, and immediately after tracheal intubation.

Also, ankle-brachial blood pressure differences measured in the beach chair position were significantly higher than anklebrachial blood pressure differences measured immediately before the beach chair position. These results suggest that brachial blood pressure should be measured in the beach chair position. If it is not possible to measure brachial blood pressure before the beach chair position or in the beach chair position, brachial blood pressure could be inferred using the regression equations of the present study (Table 2).

In the situation that the ankle blood pressure is higher than brachial blood pressure, if anesthesiologists regard ankle blood pressure as equal to blood pressure measured at the heart level, they would increase anesthetic agent concentrations in order to decrease ankle blood pressure. If brachial blood pressure is lower even though ankle blood pressure measured higher, and if brain blood pressure falls below the lower limit of autoregulation, misinterpretation of the ankle blood pressure measurement might result in cerebral ischemia and several anesthetic complications. Deep general anesthesia can depress brain function, and postoperative cognitive dysfunction could persist for several months in elderly patients subjected to deep general anesthesia $[13,14]$. In order to reduce anesthetic complications due to deep anesthesia, anesthesiologists should consider the ankle-brachial blood pressure difference when monitoring anesthesia using ankle blood pressure.

In the beach chair position, anesthesia-related complications, including spinal and cerebral ischemia and death are more common than in any other positions $[3,15,16]$. In the previous case that the left shoulder replacement surgery was performed in the beach chair position and a noninvasive blood pressure cuff was placed on the calf, brain death was reported. In that case report, the calf blood pressure was not compared with the left arm blood pressure [3]. Also, no arterial catheter during anesthesia was placed although deliberately induced hypotension was used. The calf blood pressure in that case report was continuously decreased to $50 / 25 \mathrm{mmHg}$. It is known that the magnitude of the gradient is approximately 2 $\mathrm{mmHg}$ per inch of height differential $(0.77 \mathrm{mmHg}$ decrease for every centimeter gradient) $[3,17,18]$. If height between brain and calf were approximately $45 \mathrm{~cm}$ in that case report, brain blood pressure might be lower by approximately 34.65 (0.77 $\times 45) \mathrm{mmHg}$ than the calf blood pressure. At that instant that the calf blood pressure was $50 / 25 \mathrm{mmHg}$, brain systolic blood pressure might be approximately 15.35 (50 minus 34.65 ) $\mathrm{mmHg}$ that indicates severely compromised cerebral perfusion. This severely compromised brain systolic blood pressure might result in irreversible brain death.

In the beach chair position, anesthesiologists must take care to ensure an adequate cerebral perfusion [17]. Although autoregulation normally maintains a constant cerebral blood flow between mean arterial pressure $50 \mathrm{mmHg}$ and $150 \mathrm{mmHg}$, cerebral blood flow can be compromised below a critical cerebral perfusion pressure that indicates lower autoregulatory limit. In the beach chair position, the blood pressure at the ankle is significantly higher than at the head or arm. In the sitting position, there exists a significant hydrostatic gradient between the brain and the site of blood pressure measurement. In the present study in which the cuff was placed on the patient's brachium and ankle, height between brain and brachium was approximately $23 \mathrm{~cm}$ in $150 \mathrm{~cm}$ tall female or $30 \mathrm{~cm}$ in $178 \mathrm{~cm}$ tall male. Therefore, brain blood pressure might be lower by approximately $18(0.77 \times 23)$ or 23 $(0.77 \times 30) \mathrm{mmHg}$ than brachial blood pressure. Also, because height between brachium and ankle in the present study was approximately $28 \mathrm{~cm}$ in $150 \mathrm{~cm}$ tall female or $30 \mathrm{~cm}$ in $178 \mathrm{~cm}$ tall male, it is predicted that brachial blood pressure will be lower by approximately $22(0.77 \times 28)$ or $23(0.77 \times 30) \mathrm{mmHg}$ than ankle blood pressure. These calculated values (22 or 23 $\mathrm{mmHg}$ ) were similar to the ankle-brachial difference of mean blood pressure of the present study in the beach chair position $(23.32 \pm 7.52$, Table 1$)$, although these values were different to the ankle-brachial blood difference of systolic blood pressure (33.97 $\pm 10.67 \mathrm{mmHg})$. The brachial mean blood pressure was lower by approximately $23 \mathrm{mmHg}$ than ankle mean blood pressure in the beach chair position. On considering these facts, a mean arterial pressure of $70 \mathrm{mmHg}$ measured at the ankle might result in unacceptably low cerebral perfusion pressures [17].

When arthroscopic shoulder surgery was done in the beach chair position, anesthesiologist must consider invasive arterial blood pressure monitoring with the transducer at the level of the external meatus. Also, anesthesiologist must consider the risks associated with invasive blood pressure monitoring [19]. If patients have significant risk factors for cerebrovascular disease, invasive arterial blood pressure monitoring would be needed. If arthroscopic shoulder surgery is performed in patients without significant risk factors for cerebrovascular disease, brachial blood pressures should be measured in the operations of shoulder and upper extremity even if the intravenous line is 
in the unoperated upper extremity. If brachial blood pressures are measured, anesthetic complications that can occur during shoulder surgery in the beach chair position might be prevented.

In the event that brachial systolic blood pressure cannot be measured in the beach chair position, brachial systolic blood pressure could be inferred from ankle systolic blood pressure using the regression equations described in Table 2 .

1. If brachial systolic blood pressure cannot be measured in the beach chair position, and if ankle systolic blood pressure is $135 \mathrm{mmHg}$ in the beach chair position, it may be predicted that the brachial systolic blood pressure in the beach chair position will be approximately $100.98 \mathrm{mmHg}$ (Brachial SBP in Beach $=-13.77+0.85$ ankle SBP in Beach $=-13.77+0.85 \times 135=$ 100.98).

2. Brachial systolic blood pressure in the beach chair position may be predicted if the SABBPD is measured before the beach chair position. If the SABBPD before the beach chair position is 20 , and if ankle systolic blood pressure is $134 \mathrm{mmHg}$ in the beach chair position, then the brachial systolic blood pressure in the beach chair position will be approximately 100.31 (134 minus 33.69) $\mathrm{mmHg}$ (Brachial SABBPD in Beach $=23.89+0.49$ SABBPD in BeBeach $=23.89+0.49 \times 20=33.69$ ).

In conclusion, ankle blood pressure was significantly higher than brachial blood pressure before induction of anesthesia, during anesthetic induction, immediately after tracheal intubation, before the beach chair position, and in the beach chair position. These results suggest that anesthesiologists should consider these ankle-brachial blood pressure differences in monitoring anesthesia in the beach chair position.

\section{Acknowledgments}

The authors thank the residents of the Department of Anesthesiology and Pain Medicine and the nurses of the postanesthetic care unit of Wonju Christian Hospital, Wonju, Gwangwon-Do, South Korea, for helping with the data sampling.

\section{References}

1. Wax DB, Lin HM, Leibowitz AB. Invasive and concomitant noninvasive intraoperative blood pressure monitoring: observed differences in measurements and associated therapeutic interventions. Anesthesiology 2011; 115: 973-8.

2. Park TS, Kim YS. Neuropraxia of the cutaneous nerve of the cervical plexus after shoulder arthroscopy. Arthroscopy 2005; 21: 631.
3. Pohl A, Cullen DJ. Cerebral ischemia during shoulder surgery in the upright position: a case series. J Clin Anesth 2005; 17: 463-9.

4. Skyhar MJ, Altchek DW, Warren RF, Wickiewicz TL, O'Brien SJ. Shoulder arthroscopy with the patient in the beach-chair position. Arthroscopy 1988; 4: 256-9.

5. Bhatti MT, Enneking FK. Visual loss and ophthalmoplegia after shoulder surgery. Anesth Analg 2003; 96: 899-902.

6. Gale T, Leslie K. Anaesthesia for neurosurgery in the sitting position. J Clin Neurosci 2004; 11: 693-6.

7. Choi JC, Yoon KB, Um DJ, Kim C, Kim JS, Lee SG. Intravenous magnesium sulfate administration reduces propofol infusion requirements during maintenance of propofol-N2O anesthesia: part I: comparing propofol requirements according to hemodynamic responses: part II: comparing bispectral index in control and magnesium groups. Anesthesiology 2002; 97: 1137-41.

8. Bromm B, Scharein E, Vahle-Hinz C. Cortex areas involved in the processing of normal and altered pain. Prog Brain Res 2000; 129: 289-302.

9. Beattie C. History and principles of anesthesiology. In: Goodman \& Gilman's the Pharmacological Basis of Therapeutics. 10th ed. Edited by Hardman JG, Limbird LE, Gilman AG: New York, McGraw-Hill. 2001, pp 321-35.

10. Doobay AV, Anand SS. Sensitivity and specificity of the ankle-brachial index to predict future cardiovascular outcomes: a systematic review. Arterioscler Thromb Vasc Biol 2005; 25: 1463-9.

11. Wilkes JM, DiPalma JA. Brachial blood pressure monitoring versus ankle monitoring during colonoscopy. South Med J 2004; 97: 93941.

12. Shein-Chung C, Jun S, Hansheng W. Sample size calculations in clinical research. 2nd ed. New York, Chapman \& Hall/CRC. 2007, pp 52-4.

13. Brown EN, Lydic R, Schiff ND. General anesthesia, sleep, and coma. N Engl J Med 2010; 363: 2638-50.

14. Monk TG, Weldon BC, Garvan CW, Dede DE, van der Aa MT, Heilman KM, et al. Predictors of cognitive dysfunction after major noncardiac surgery. Anesthesiology 2008; 108: 18-30.

15. Marecek GS, Saltzman MD. Complications in shoulder arthroscopy. Orthopedics 2010; 33: 492-7.

16. Papadonikolakis A, Wiesler ER, Olympio MA, Poehling GG. Avoiding catastrophic complications of stroke and death related to shoulder surgery in the sitting position. Arthroscopy 2008; 24: 4812.

17. Rains DD, Rooke GA, Wahl CJ. Pathomechanisms and complications related to patient positioning and anesthesia during shoulder arthroscopy. Arthroscopy 2011; 27: 532-41.

18. Sia S. Hypotensive technique and sitting position in shoulder surgery. Anesth Analg 2003; 97: 1198.

19. Brzezinski M, Luisetti T, London MJ. Radial artery cannulation: a comprehensive review of recent anatomic and physiologic investigations. Anesth Analg 2009; 109: 1763-81. 